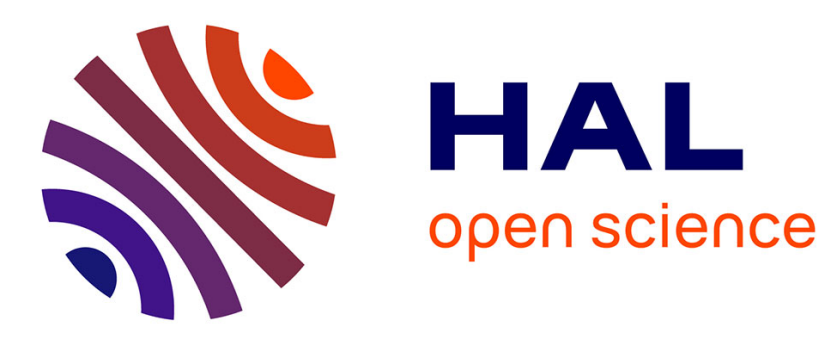

\title{
Humanoid motion generation and swept volumes: theoretical bounds for safe steps
}

Nicolas Perrin, Olivier Stasse, Florent Lamiraux, Eiichi Yoshida

\section{To cite this version:}

Nicolas Perrin, Olivier Stasse, Florent Lamiraux, Eiichi Yoshida. Humanoid motion generation and swept volumes: theoretical bounds for safe steps. Advanced Robotics, 2013, 27 (14), pp.1045 - 1058. 10.1080/01691864.2013.805468 . hal-01113510

\section{HAL Id: hal-01113510 https://hal.science/hal-01113510}

Submitted on 5 Feb 2015

HAL is a multi-disciplinary open access archive for the deposit and dissemination of scientific research documents, whether they are published or not. The documents may come from teaching and research institutions in France or abroad, or from public or private research centers.
L'archive ouverte pluridisciplinaire HAL, est destinée au dépôt et à la diffusion de documents scientifiques de niveau recherche, publiés ou non, émanant des établissements d'enseignement et de recherche français ou étrangers, des laboratoires publics ou privés. 


\title{
Humanoid motion generation and swept volumes: theoretical bounds for safe steps
}

\author{
Nicolas Perrin $\quad$ Olivier Stasse Florent Lamiraux Eiichi Yoshida \\ N. Perrin is with Department of Advanced Robotics, Istituto Italiano di Tecnologia, via Morego, 30,16163 \\ Genova, Italy. nicolas.perrin@iit.it \\ O. Stasse and F. Lamiraux are with CNRS/LAAS, Université de Toulouse UPS, INSA, INP, ISAE, 7 avenue \\ du colonel Roche, F-31077 Toulouse, France. \{ostasse,florent\}@laas.fr \\ E. Yoshida is with CNRS-AIST Joint Robotics Laboratory, UMI3218/CRT, Tsukuba, Japan. \\ e.yoshida@aist.go.jp
}

\begin{abstract}
In the context of humanoid robot footstep planning based on a continuous action set, we conduct an analysis of the sensitivity of a walking pattern generator. Given a variation of an input vector, we calculate a bound on the variation of the volume swept by the robot lower body during the corresponding actions (steps). Since the input vector depends on real parameters, there is an infinite number of possible steps, but the calculated bound permits a sound and safe use of only a finite number of swept volumes to account for all the possible motions. After numerical evaluations, we discuss potential applications.
\end{abstract}

\section{Introduction}

Humanoid robot footstep planning based on a discrete action set (i.e. with a finite set of possible steps) has been extensively studied over the past decade, and has led to interesting applied results (see for example $[14,3,4,5,10])$. Recently however, an increasing interest has been drawn to footstep planning based on continuous action sets, because it can lead to smaller sequences of steps and can increase the capacity to avoid obstacles (see $[7,8,1,17]$ ). As it is easier to deal with continuous sets when they have few dimensions, in a previous work ([16]), we introduced a walking pattern generator which produces half-steps with an input space of only 3 dimensions.

The use of swept volumes is widespread in robotics, especially for path planning $([18,11])$, but relatively absent in the field of humanoid robotics, where, for the sake of computational efficiency, simpler bounding volumes are often preferred $([21,9])$.

In [15], we used the walking pattern generator of [16] and swept volume approximations to obtain fast motion planning with precise 3D obstacle avoidance (the swept volume approximations have a tree 

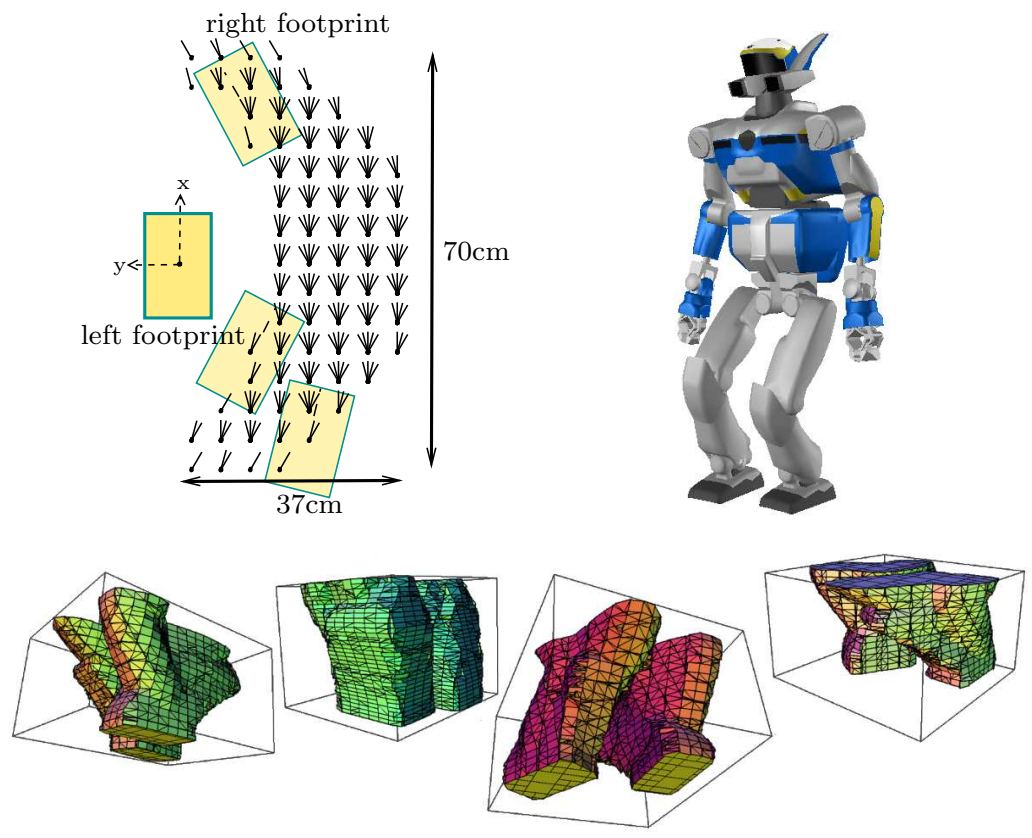

Figure 1: In [15], we used a finite action set of 276 points, and for each input we approximated the volume swept by a part of the robot lower body during the corresponding half-step. On the top left of the figure, the points and small line segments represent all the possible starting and ending positions and orientations of the swing foot for the steps in this finite action set. The 3D polygons represent some of the swept volume approximations obtained for these steps.

structure which is used to reduce the time spent in collision checking). Unfortunately, since only a finite number of swept volume approximations can be built in finite time, we had to use a discrete action set (called transition model in [15]): each action corresponds to a step, and the corresponding swept volume is approximated offline, in order to be quickly usable online (see Figure 1). In the present paper, our goal is to explore the possibility of using the same technique with a continuous action set, following this simple idea: if we would expand the swept volumes by a fixed margin, then they could cover neighborhoods of actions instead of single actions, and we could use a finite number of swept volumes in order to cover the whole continuous input space of actions, while keeping the soundness of collision checks. Before doing this it is fundamental to know the relation between the size of the margin and the size of the neighborhoods. In other words, we need to know how many swept volumes are needed in order to cover the whole input space when the margin has a fixed size $\epsilon$. This is the question we answer in this paper (with Theorem 5.1).

\section{Problem statement}

The walking pattern generator in [16] works as follows: it first produces a collision-free sequence of isolated "half-steps" concatenated with zero-speed connections, and then, online, it uses a simple and 


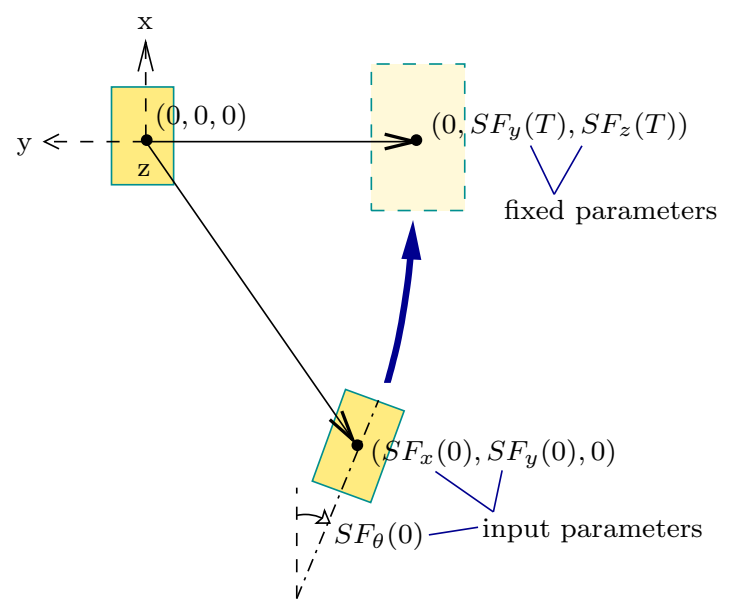

Figure 2: Here we show an upward half-step seen from above. It is fully determined by the 5 parameters $S F_{x}(0), S F_{y}(0), S F_{\theta}(0), S F_{y}(T)$ and $S F_{z}(T)$. The three first are the input parameters, and the two last are fixed. $S F_{y}(T)$ and $S F_{z}(T)$ define the "via-point configuration" and were chosen according to the robot dimensions so that to give to its steps a reasonable clearance.

efficient homotopy to smooth the sequence into a more fluid walking motion. The swept volume approximations are used with the isolated half-steps in order to speed up the online collisions checks. There are two types of half-steps: upward and downward. An upward half-step consists in rising the swing foot from the ground to reach an intermediate fixed "via-point configuration" (like in [14]). Conversely a downward half-step starts in the "via-point configuration" and reaches (with zero speed) a configuration where the swing foot lies on the ground. We denote by CoM the center of mass of the whole robot and assume that it is rigidly linked with the waist of the robot (a classical assumption for walking pattern generators). Through inverse position kinematics, both upward and downward half-steps are completely defined by the horizontal trajectory of the CoM (it stays at a fixed height), the waist orientation in function of the time, and the swing foot position and orientation trajectories. Using some arbitrary and convenient assumptions, these trajectories can be generated with only 3 input parameters: the position and orientation of the swing foot when it is on the ground.

In this paper we make a convenient assumption that was not made in [16] or [15]: we suppose that at all time, the orientation of the waist is equal to the average of the foot orientations. Because of this and an additional symmetry properties of the functions used to produce the trajectories (equations (6)), upward half-steps and downward half-steps are completely symmetric: a downward half-step is just an upward half-step with time going backwards. As a result, we don't need to consider both downward and upward half-steps: the bound obtained for upward half-steps will directly apply to downward halfsteps. Besides, the half-steps with left and right support foot are also symmetric, so in the end we only need to consider upward half-steps with left support foot. They are defined as shown on Figure 2: a fixed frame $(\mathrm{x}, \mathrm{y}, \mathrm{z})$ is attached to the ankle joint of the support foot, the $\mathrm{x}$-axis defining the reference orientation (equal to the support foot orientation). At the end of the half-step, $t=T$ ( $T$ is fixed and 
doesn't depend on the input parameters), the robot is in the fixed "via-point configuration": the swing foot coordinates are $\left(0, S F_{y}(T), S F_{z}(T)\right)$, and its orientation is zero. The input parameters are $S F_{x}(0)$, $S F_{y}(0)$, and $S F_{\theta}(0)$ : the initial position and orientation of the swing foot. Thanks to a few arbitrary restrictions (including the one on the waist orientation), these input parameters completely set the 6 following functions of the time, which entirely define the lower body trajectory through inverse position kinematics:

- the waist horizontal position, which is equal to the CoM horizontal position (the waist and CoM are rigidly linked) : $x(t), y(t)$

- the swing foot position: $S F_{x}(t), S F_{y}(t), S F_{z}(t)$

- the swing foot orientation $S F_{\theta}(t)$

We define the robot lower body as the union of the two legs: the two thighs, calves and feet. For a given vector of input parameters $\left(S F_{x}(0), S F_{y}(0), S F_{\theta}(0)\right)$, we denote by $\mathcal{S} \mathcal{V}\left(\left(S F_{x}(0), S F_{y}(0), S F_{\theta}(0)\right)\right)$ the volume swept by the robot lower body during the corresponding half-step. We also denote by $\mathcal{E}$ the continuous action set, i.e. the set of input vectors $\vec{v}$ such that the corresponding half-step is feasible on a flat ground without obstacles (no self-collision, no joint limits violations -we mention in section 4 some of the restrictions imposed on the joint limits-, ...).

The question we want to answer is related to the following one:

Given an input vector $\left(S F_{x}(0), S F_{y}(0), S F_{\theta}(0)\right) \in \mathcal{E}$, and a small variation $(\Delta x, \Delta y, \Delta \theta)$ such that $\left(S F_{x}(0)+\Delta x, S F_{y}(0)+\Delta y, S F_{\theta}(0)+\Delta \theta\right)$ also belongs to $\mathcal{E}$, how can we bound, in function of $(\Delta x, \Delta y, \Delta \theta)$, the variation that it implies for the swept volume?

In other words:

Given two input vectors $\left(S F_{x}(0), S F_{y}(0), S F_{\theta}(0)\right) \in \mathcal{E}$ and $\left(S F_{x}(0)+\Delta x, S F_{y}(0)+\Delta y, S F_{\theta}(0)+\Delta \theta\right) \in$ $\mathcal{E}$, how can we bound, in function of $(\Delta x, \Delta y, \Delta \theta)$, the Hausdorff distance between $\mathcal{S} \mathcal{V}\left(\left(S F_{x}(0)\right.\right.$, $\left.\left.S F_{y}(0), S F_{\theta}(0)\right)\right)$ and $\mathcal{S V}\left(\left(S F_{x}(0)+\Delta x, S F_{y}(0)+\Delta y, S F_{\theta}(0)+\Delta \theta\right)\right)$ ?

We answer this question by dividing the problem into three parts: first, in section 3 , we will bound the variation induced on the waist and foot trajectories by a slight modification of the input parameters. Then, in section 4, we will bound the displacement of the physical points of the robot leg induced by a slight modification of the end effector (the foot) configuration in the workspace. Finally we combine the two results in section 5 and obtain the main theorem (Theorem 5.1). In sections 6 and 7, we present numerical results and conclude.

\section{Sensitivity of the trajectories generation}

Let us consider an upward half-step defined by the 3 parameters $S F_{x}(0), S F_{y}(0)$ and $S F_{\theta}(0)$. As mentioned in the previous section, these parameters are used to set the following functions of the time: 
- the waist horizontal position, which is equal to the CoM horizontal position (the waist and CoM are rigidly linked) : $x(t), y(t)$

- the swing foot position: $S F_{x}(t), S F_{y}(t), S F_{z}(t)$

- the swing foot orientation $S F_{\theta}(t)$

For all these trajectories except $x(t)$ and $y(t)$, we use $C^{2}$ cubic splines. $S F_{z}(t)$ does not depend on the input parameters. For the other functions $S F_{x}(t), S F_{y}(t)$ and $S F_{\theta}(t)$, we use a single operator $\mathcal{F}$ which maps a couple of reals $(a, b)$ to a $C^{2}$ cubic spline $s(t)$ which smoothly goes from $a$ at $t=0$ to $b$ at $t=T$ with constraints such as zero speed at $t=0$ and $t=T$ :

$$
\begin{gathered}
S F_{x}(t)=\mathcal{F}\left(S F_{x}(0), 0\right)(t) \\
S F_{y}(t)=\mathcal{F}\left(S F_{y}(0), S F_{y}(T)\right)(t) \\
S F_{\theta}(t)=\mathcal{F}\left(S F_{\theta}(0), 0\right)(t)
\end{gathered}
$$

$\mathcal{F}$ has three important properties:

$$
\begin{gathered}
\mathcal{F}(a+b, c+d)=\mathcal{F}(a, c)+\mathcal{F}(b, d) \\
\forall t \in[0, T], \min (a, b) \leq \mathcal{F}(a, b)(t) \leq \max (a, b) \\
\forall t \in[0, T], \mathcal{F}(a, b)(t)=\mathcal{F}(b, a)(T-t)
\end{gathered}
$$

If we call $x^{\prime}, y^{\prime}, S F_{x}^{\prime}, S F_{y}^{\prime}$ and $S F_{\theta}^{\prime}$ the trajectories associated to the input parameters $\left(S F_{x}(0)+\right.$ $\left.\Delta x, S F_{y}(0)+\Delta y, S F_{\theta}(0)+\Delta \theta\right)$, then from the above properties of $\mathcal{F}$ follows, for all $t \in[0, T]$ :

$$
\begin{aligned}
& \min (0, \Delta x) \leq S F_{x}^{\prime}(t)-S F_{x}(t) \leq \max (0, \Delta x) \\
& \min (0, \Delta y) \leq S F_{y}^{\prime}(t)-S F_{y}(t) \leq \max (0, \Delta y) \\
& \min (0, \Delta \theta) \leq S F_{\theta}^{\prime}(t)-S F_{\theta}(t) \leq \max (0, \Delta \theta)
\end{aligned}
$$

Now we will try to obtain the same inequalities for $x$ and $y$. $x$ and $y$ are actually generated from the trajectory of a virtual point on the ground: the Zero Moment Point (ZMP, see [20]). We denote its coordinates by $\left(p_{x}(t), p_{y}(t)\right)$. Our walking pattern generator is based on a classical simplified model of the robot dynamics (the Linear Inverted Pendulum Mode, see [12]), where the ZMP trajectories $p_{x}, p_{y}$ and the CoM trajectories $x, y$ are linked by the following equations:

$$
\begin{gathered}
p_{x}=Z(x) \\
p_{y}=Z(y) \\
\text { with } Z \triangleq I d-\frac{z_{c}}{g} \frac{d}{d t^{2}},
\end{gathered}
$$

where $z_{c}$ is the fixed height of the CoM and $g$ the gravity of Earth. 


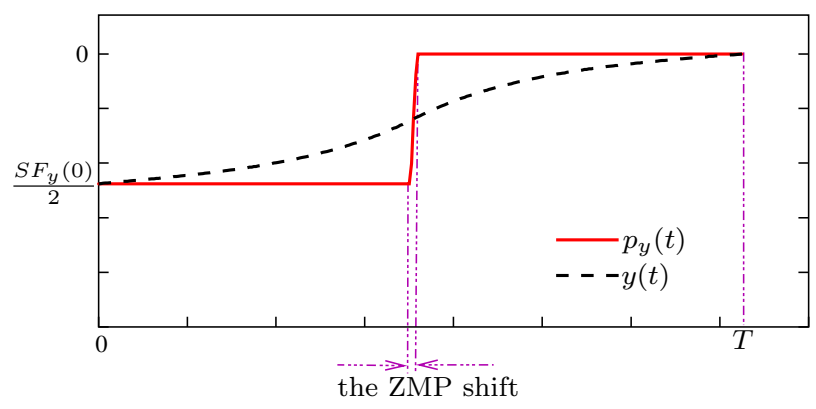

Figure 3: We consider the upward half-step of Figure 2, and show the ZMP trajectory along the y-axis: $p_{y}(t)$, as well as the corresponding CoM trajectory: $y(t)$.

We require that $p_{x}(0)=x(0)=\frac{S F_{x}(0)}{2}$ and $p_{y}(0)=y(0)=\frac{S F_{y}(0)}{2}$, and $p_{x}(T)=x(T)=0$ and $p_{y}(T)=y(T)=0$ (i.e. both the CoM and ZMP start right between the feet and end above the center of the support foot). It follows that if $p_{x}$ and $p_{y}$ are fixed as $C^{2}$ cubic splines, there is a unique $C^{4}$ solution for $x$ and $y$ over $[0, T]$ (in which $\dot{x}(0), \dot{y}(0)$ and $\dot{x}(T), \dot{y}(T)$ are not zero, but, as shown in [16], can be neglected if $T$ is large enough and $p_{x}$ and $p_{y}$ well chosen).

In order to generate $p_{x}$ and $p_{y}$, we use an operator $\mathcal{F}_{Z}$ which maps a couple of reals $(a, b)$ to a $C^{2}$ cubic spline which quickly shifts from $a$ to $b$ around time $t=T / 2$ (see Figure 3 ). $\mathcal{F}_{Z}$ verifies the same properties (4), (5) and (6) as $\mathcal{F}$. We have:

$$
\begin{aligned}
& p_{x}^{\prime}=\mathcal{F}_{Z}\left(\frac{S F_{x}(0)+\Delta x}{2}, 0\right)=p_{x}+\mathcal{F}_{Z}\left(\frac{\Delta x}{2}, 0\right) \\
& p_{y}^{\prime}=\mathcal{F}_{Z}\left(\frac{S F_{y}(0)+\Delta y}{2}, 0\right)=p_{y}+\mathcal{F}_{Z}\left(\frac{\Delta y}{2}, 0\right)
\end{aligned}
$$

Using the linearity of operator $Z$, we can show that if for any $C^{2}$ cubic splines $p_{x}$ and $p_{y}$ we denote by $Z^{-1}\left(p_{x}\right)$ and $Z^{-1}\left(p_{y}\right)$ the unique $C^{4}$ solutions for the CoM trajectories, then we have also:

$$
\begin{aligned}
& x^{\prime}=Z^{-1}\left(p_{x}^{\prime}\right)=x+Z^{-1}\left(\mathcal{F}_{Z}(\Delta x / 2,0)\right) \\
& y^{\prime}=Z^{-1}\left(p_{y}^{\prime}\right)=y+Z^{-1}\left(\mathcal{F}_{Z}(\Delta y / 2,0)\right)
\end{aligned}
$$

Now let's have look on the properties of this operator $Z^{-1}$. Since the result is a continuous function, as shown on Figure 4, the equations (10), (11), (12) and the restrictions $Z^{-1}(p)(0)=p(0), Z^{-1}(p)(T)=$ $p(T)$ imply that for a $C^{2}$ cubic spline $p$ over $[0, T]$, we necessarily have:

$$
\forall t \in[0, T], \min _{\tau \in[0, T]}(p(\tau)) \leq Z^{-1}(p)(t) \leq \max _{\tau \in[0, T]}(p(\tau))
$$

Since we also have for all $t \in[0, T], \mathcal{F}_{Z}(\Delta x / 2,0)(t) \leq \max (0, \Delta x / 2), \mathcal{F}_{Z}(\Delta x / 2,0)(t) \geq \min (0, \Delta x / 2)$ and the same for $\mathcal{F}_{Z}(\Delta y / 2,0)(t)$, we finally obtain the inequalities we were looking for: for all $t \in[0, T]$,

$$
\begin{aligned}
& \min \left(0, \frac{\Delta x}{2}\right) \leq x^{\prime}(t)-x(t) \leq \max \left(0, \frac{\Delta x}{2}\right) \\
& \min \left(0, \frac{\Delta y}{2}\right) \leq y^{\prime}(t)-y(t) \leq \max \left(0, \frac{\Delta y}{2}\right)
\end{aligned}
$$




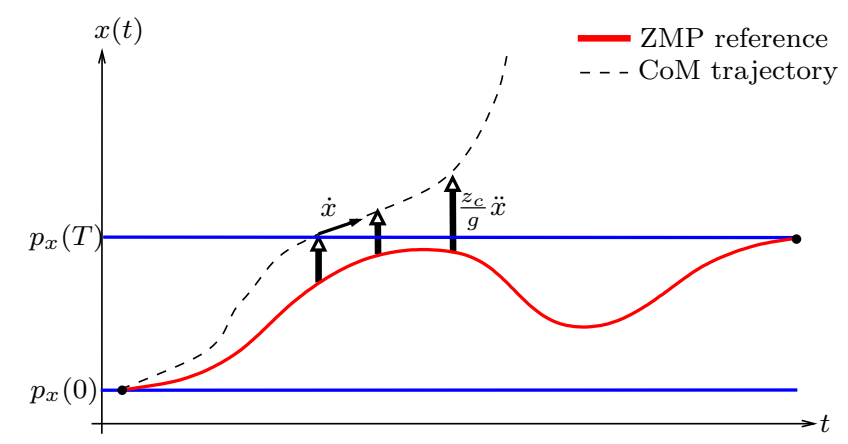

Figure 4: If one of the components of the CoM position, say for example $x(t)$, goes outside the stripe $\left[p_{x}(0), p_{x}(T)\right]$ defined by the ZMP reference, then the acceleration $\ddot{x}(t)$, obtained with the equation (12), can only make $x(t)$ diverge. Therefore $x(t)$ must stay inside the stripe for all $t \in[0, T]$.

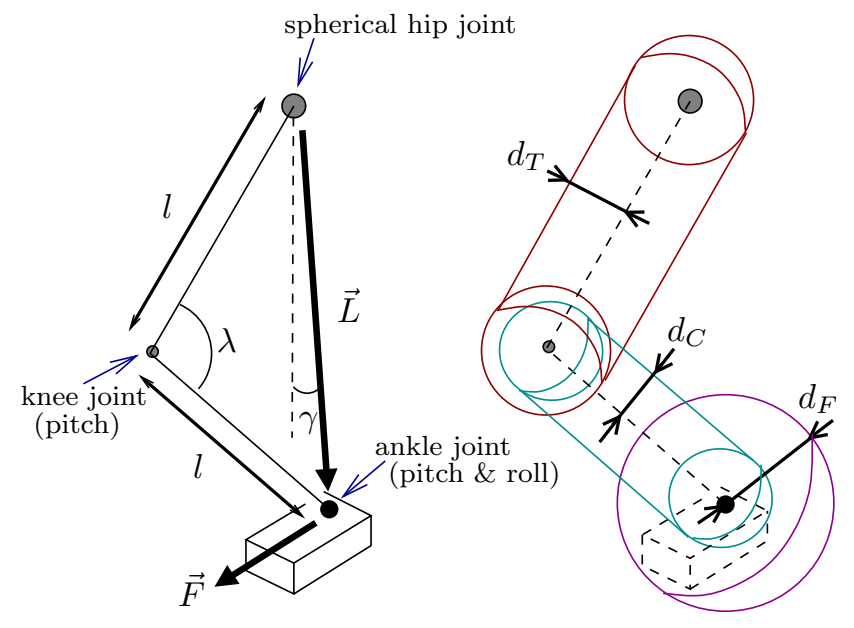

Figure 5: A simple 6-DOF robot leg.

\section{Sensitivity of the inverse kinematics of a simple humanoid robot leg}

In this section we consider a very generic robot leg with 6 degrees of freedom. Its geometry is exactly similar or almost similar to the geometry of the legs of several humanoid robots, including ASIMO, HRP-2, HRP-4, QRIO, HUBO, WABIAN, and LOLA. The robot leg consists in 3 joints: the hip (yaw + pitch + roll), the knee (only pitch), and the ankle $($ pitch + roll), the foot being the end effector. The center of the hip joint is the origin $(0,0,0)$ of the workspace, and an horizontal reference orientation is fixed. Since we will assume that the foot always stays parallel to the ground (which is assumed to be horizontal), as shown on Figure 5, the configuration of the leg is entirely defined by two vectors: $\vec{L}$ (the vector from the hip joint to the ankle joint), and $\vec{F}$, a unit vector defining the orientation of the foot. $\vec{F}$ is supposed to be always included in the plane defined by the hip, knee and ankle. The results proven in this section directly apply only if this specific organization is verified, which is common in walking robots. However, for slightly different geometries, the proofs can be adapted, and lead to bounds of the 
same order of magnitude. $\vec{F}$ is also completely defined by its orientation in the horizontal plane, i.e. by a single angle value $\theta$. The leg is composed of three bodies: the thigh, the calf, and the foot. For the thigh and the calf, we assume that all the physical points of the robot are contained in a volume defined by 2 spheres and a cylinder: see Figure 5 . The spheres and cylinder of the thigh have radius $d_{T}$, while the spheres and cylinder of the calf have radius $d_{C}$. The two links and cylinders have the same length $l$. We also assume that the foot is entirely contained in a sphere of radius $d_{F}$ and center the ankle joint. We impose a few restrictions on the joint values: let us call $\nu$ the vertical axis passing through $(0,0,0), \gamma$ the angle between $\nu$ and $\vec{L}$, and $\lambda$ the value of the knee joint (see Figure 5 ). The restrictions are: $0 \leq \gamma \leq \gamma_{\max }<\pi / 3$, and $0<\lambda_{\min } \leq \lambda \leq \lambda_{\max }<\pi$. Here is the context of our problem: the current configuration of the leg is $(\vec{L}, \theta)$ (where $\theta$ is the orientation of $\vec{F}$ ), and we modify it to $(\vec{L}+\overrightarrow{\Delta L}, \theta+\Delta \theta)$, with $\overrightarrow{\Delta L}$ and $\Delta \theta$ "relatively small" (we will discuss about this notion later). We ask the following question: how can we bound, in function of $\overrightarrow{\Delta L}$ and $\Delta \theta$ only, the distance between any physical point of the leg in configuration $(\vec{L}, \theta)$, and the same physical point when the configuration is $(\vec{L}+\overrightarrow{\Delta L}, \theta+\Delta \theta)$ ?

Even a closed-form solution of the inverse kinematics of the leg (see [19], section 1.7.1) cannot be straightforwardly used to obtain a tight bound, and the same is true for an approach based on the inverse Jacobian, so instead we chose to use purely geometrical considerations in an attempt to obtain a tighter bound, and reached the following theorem:

Theorem 4.1. For any point of the robot leg, the displacement resulting from the motion from configuration $(\vec{L}, \theta)$ to configuration $(\vec{L}+\overrightarrow{\Delta L}, \theta+\Delta \theta)$ is bounded by the maximum of the three following expressions:

$$
\begin{array}{ll}
1) & 2 \sin \left(\frac{\alpha_{\max }}{2}\right)\left(l+\max \left(d_{T}, d_{C}\right)\right) \\
& +\sin \left(\frac{1}{2} \arcsin \left(\frac{2 \sin \left(\alpha_{\max } / 2\right)}{\cos \gamma_{\max }}\right)\right)\left(l+\max \left(d_{T}, d_{C}\right)\right) \\
& +2 \sin \left(\beta_{\max } / 2\right)\left(l+\max \left(d_{T}, d_{C}\right)\right) \\
& +2 \sin \left(\frac{\cos \gamma_{\max } \cdot|\Delta \theta|}{4 \cos \gamma_{\max }-2}\right)\left(l \cdot \cos \left(\frac{\lambda_{\min }}{2}\right)+\max \left(d_{T}, d_{C}\right)\right) \\
2) \quad & \|\overrightarrow{\Delta L}\|+2 \sin \left(\alpha_{\max } / 2\right) d_{C} \\
& +2 \sin \left(\frac{1}{2} \arcsin \left(\frac{2 \sin \left(\alpha_{\max } / 2\right)}{\cos \gamma_{\max }}\right)\right) d_{C} \\
& +2 \sin \left(\beta_{\max } / 2\right) d_{T}+2 \sin \left(\frac{\cos \gamma_{\max } \cdot|\Delta \theta|}{4 \cos \gamma_{\max }-2}\right) d_{C} \\
\text { 3) } & \|\overrightarrow{\Delta L}\|+2 \sin \left(\frac{|\Delta \theta|}{2}\right) d_{F}
\end{array}
$$

with:

$$
\left\{\begin{array}{c}
\alpha_{\max }=\arcsin \left(\frac{\|\overrightarrow{\Delta L}\|}{2 l \sin \left(\frac{\lambda_{\min }}{2}\right)}\right) \\
\beta_{\max }=\frac{\lambda_{\max }}{2}-\arcsin \left(\sin \left(\frac{\lambda_{\max }}{2}\right)-\frac{\|\overrightarrow{\Delta L}\|}{2 l}\right)
\end{array}\right.
$$

We denote by $\mathcal{B}(\|\overrightarrow{\Delta L}\|,|\Delta \theta|)$ this bound.

We will decompose the demonstration of this theorem in several steps, and start with a preliminary lemma which shows that if we can bound the displacement undergone by the points inside the 5 spheres previously defined ( 2 spheres for thigh, 2 spheres for the calf, and 1 for the foot), we will have obtained a global bound that applies to any physical point of the robot leg (in other terms, no need to consider 
the cylinders). The proofs of these steps use only basic mathematics but can be quite complex, so most of the elements of the proofs are in appendix. During the demonstrations we will make some assumptions that have not been stated before. We don't prove it here, but it can be verified that all those assumptions are true when $\|\overrightarrow{\Delta L}\|$ and $\overrightarrow{\Delta \theta}$ are relatively small. We don't give more details because as $\|\overrightarrow{\Delta L}\|$ and $\overrightarrow{\Delta \theta}$ are always very small in the potential applications, these assumptions always hold in practice.

Lemma 4.1. Let's consider the robot leg whose bodies are exactly the unions of the spheres and cylinders previously defined. For any motion of this leg, the maximum displacement undergone by its physical points is obtained for a point inside one of the spheres.

Proof. Thanks to Chasles' Screw displacement theorem (see [2], section 3.11), we know that the transformation undergone by any rigid body of the robot leg can be written as the commutative composition of a rotation about an axis and a translation along the same axis. Since the displacements resulting from the rotation and the translation are orthogonal, we can take them into account separately. The translation results in the same displacement for all the points, so in order to find the maximum displacement we just need to consider the rotation. Let us project the rigid body onto a plane orthogonal to the rotation axis, and call $O$ the intersection point between the axis and the plane. We use $O$ as the origin of this plane and use vectors to represent projections of the rigid body points. Let $\vec{p}$ be such a projection; it lies on a segment line whose extremities $\vec{a}$ and $\vec{b}$ are projections of points inside the spheres at the extremities of the link. We thus have $\vec{p}=\mu \vec{a}+(1-\mu) \vec{b}$, with $\mu \in[0,1]$. If we denote by Rot the rotation, the displacement $d(\vec{p})$ undergone by the point projected on $\vec{p}$ can be written:

$$
\begin{aligned}
d(\vec{p}) & =\|\operatorname{Rot}(\vec{p})-\vec{p}\| \\
& =\|\mu(\operatorname{Rot}(\vec{a})-\vec{a})+(1-\mu)(\operatorname{Rot}(\vec{b})-\vec{b})\|
\end{aligned}
$$

Since the maximum distance from a point to a line segment is always obtained at one of its extremities, we have:

$$
\begin{gathered}
d(\vec{p}) \leq \max (\|\operatorname{Rot}(\vec{a})-\vec{a}\|,\|\operatorname{Rot}(\vec{b})-\vec{b}\|) \\
d(\vec{p}) \leq \max (d(\vec{a}), d(\vec{b}))
\end{gathered}
$$

We conclude that the maximum displacement is obtained for a point inside a sphere.

We can now start the demonstration of Theorem 4.1.

Proof. Thanks to Lemma 4.1, we know that we only need to bound the displacements for points inside the spheres, and we will naturally derive 5 bounds, one for each sphere. But first, in order to calculate these bounds, let us describe the transformations that are applied to the leg bodies.

The motion changing $(\vec{L}, \theta)$ into $(\vec{L}+\overrightarrow{\Delta L}, \theta+\Delta \theta)$ can be decomposed into three parts (see Figure 6 ): first, using hip rotations, we obtain the correct hip joint - ankle joint axis while keeping $\vec{F}$ unchanged thanks to ankle rotations; then, sliding along this axis, using the hip and knee pitch, we obtain $(\vec{L}+\overrightarrow{\Delta L}, \theta)$ (ankle rotations are again used to keep $\vec{F}$ unchanged). Finally, we use the hip joint to rotate about the 


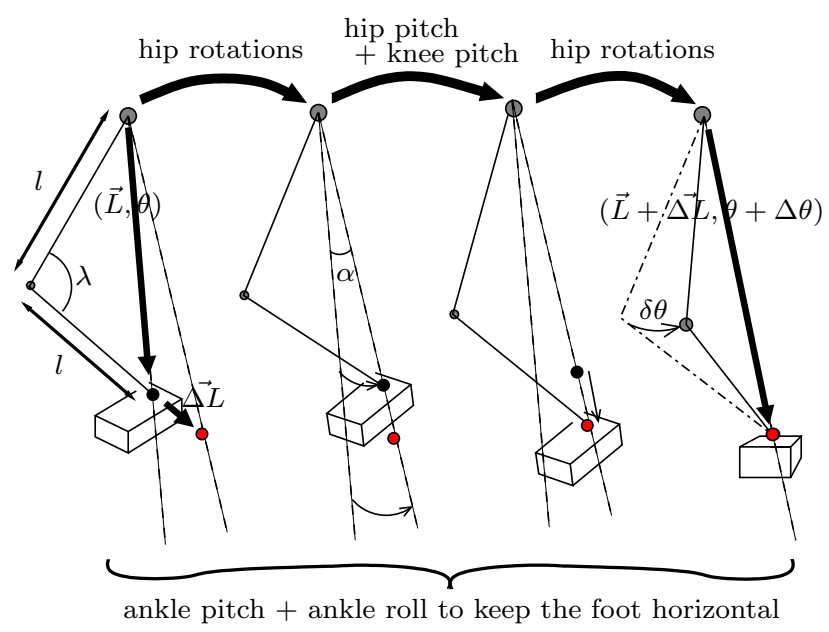

Figure 6: The three phases to go from $(\vec{L}, \theta)$ to $(\vec{L}+\overrightarrow{\Delta L}, \theta+\Delta \theta)$.

axis defined by $\vec{L}+\overrightarrow{\Delta L}$, keeping the foot parallel to the walking surface by modifying the ankle pitch and roll.

In the first phase, the correct axis can be obtained by two consecutive rotations about orthogonal axes. The first rotation of angle $\alpha_{1}$ uses only the hip pitch, and during it the foot is kept horizontal by modifying the ankle pitch. Then follows a rotation of angle $\alpha_{2}$ about the axis passing through the origin and with direction vector $\vec{F}$. During this rotation the ankle roll is used to keep the foot horizontal. So far $\vec{F}$ remains unchanged. To obtain bounds on the displacements resulting from these rotations, we will need to bound the angles $\alpha_{1}$ and $\alpha_{2}$.

In the second phase, three rotations are combined to slide the ankle along the hip joint - ankle joint axis: a rotation of angle $\beta$ (hip pitch), a rotation of angle $-2 \beta$ (knee pitch), and a rotation of angle $\beta$ (ankle pitch) to keep $\vec{F}$ unchanged (see Figure 8 in Appendix B). We will need to bound the angle $\beta$.

In the third phase, the angle $\delta \theta$ of the rotation (about the axis defined by $\vec{L}+\overrightarrow{\Delta L}$ ) is not necessarily equal to $\Delta \theta$ and needs to be bounded as well.

Since for a rotation of angle $\psi$ on a point $p$ at distance $\eta$ of the rotation axis, the resulting displacement of $p$ is $|2 \sin (\psi / 2)|$, we will rather try to bound this expression instead of bounding directly the angle value. Here are the bounds obtained for the 4 angles:

The positive angles $\alpha_{1}$ and $\alpha_{2}$ are such that:

$$
\begin{gathered}
\left|2 \sin \left(\alpha_{1} / 2\right)\right| \leq 2 \sin \left(\alpha_{\max } / 2\right) \\
\left|2 \sin \left(\alpha_{2} / 2\right)\right| \leq 2 \sin \left(\frac{1}{2} \arcsin \left(\frac{2 \sin \left(\alpha_{\max } / 2\right)}{\cos \gamma_{\max }}\right)\right)
\end{gathered}
$$

with $\alpha_{\max }=\arcsin \left(\frac{\|\vec{L}\|}{2 l \sin \left(\frac{\lambda_{\min }}{2}\right)}\right)$.

The demonstration is in Appendix A. 
The angle $\beta$ is such that:

$$
|2 \sin (|\beta| / 2)| \leq 2 \sin \left(\beta_{\max } / 2\right)
$$

with $\beta_{\max }=\frac{\lambda_{\max }}{2}-\arcsin \left(\sin \left(\frac{\lambda_{\max }}{2}\right)-\frac{\|\Delta \vec{L}\|}{2 l}\right)$.

The demonstration is in Appendix B.

\section{The angle $\delta \theta$ is such that:}

$$
|2 \sin (|\delta \theta| / 2)| \leq 2 \sin \left(\frac{\cos \gamma_{\max }}{4 \cos \gamma_{\max }-2} \cdot|\Delta \theta|\right)
$$

The demonstration is in Appendix C.

Now we can bound the displacements undergone by the points of each sphere.

\subsection{The sphere of the thigh at the hip joint}

During the first, second and third phase of the motion of the leg, this sphere undergoes 4 successive rotations of angles $\alpha_{1}, \alpha_{2}, \beta$ and $\delta \theta$, and the points of the sphere are at a distance from the rotations axes which is bounded by $d_{T}$. It follows that the total displacement undergone by any point of the sphere is bounded by the following sum:

$$
\begin{aligned}
& 2 \sin \left(\alpha_{\max } / 2\right) d_{T}+2 \sin \left(\frac{1}{2} \arcsin \left(\frac{2 \sin \left(\alpha_{\max } / 2\right)}{\cos \gamma_{\max }}\right)\right) d_{T} \\
& +2 \sin \left(\beta_{\max } / 2\right) d_{T}+2 \sin \left(\frac{\cos \gamma_{\max }}{4 \cos \gamma_{\max }-2} \cdot|\Delta \theta|\right) d_{T}
\end{aligned}
$$

\subsection{The sphere of the thigh at the knee joint}

This sphere undergoes the same rotations as the other sphere of the thigh. During the first and second phases the distance of its points to the rotation axes is bounded by $l+d_{T}$, while during the third phase, this distance is bounded by $l \cdot \cos \left(\lambda_{\min } / 2\right)+d_{T}\left(l \cdot \cos \left(\lambda_{\min } / 2\right)\right.$ is the maximum distance between the knee joint and the hip joint - ankle joint axis). It results in the following bound:

$$
\begin{aligned}
& 2\left(\sin \left(\frac{\alpha_{\max }}{2}\right)+\sin \left(\frac{1}{2} \arcsin \left(\frac{2 \sin \left(\alpha_{\max } / 2\right)}{\cos \gamma_{\max }}\right)\right)\right)\left(d_{T}+l\right) \\
& +2 \sin \left(\beta_{\max } / 2\right)\left(l+d_{T}\right) \\
& +2 \sin \left(\frac{\cos \gamma_{\max }}{4 \cos \gamma_{\max }-2} \cdot|\Delta \theta|\right)\left(l \cdot \cos \left(\lambda_{\min } / 2\right)+d_{T}\right)
\end{aligned}
$$

\subsection{The sphere of the calf at the knee joint}

This sphere undergoes the same rotations as the sphere of the thigh at the knee joint, except the rotation of angle $\beta$ : while the sphere of the thigh undergoes a rotation of angle $\beta$ about an axis at distance $l$ from its center (the axis passes through the hip joint), the sphere of the calf undergoes a rotation of angle $-\beta$ about a virtual axis also at distance $l$ (see Figure 8 in Appendix B). It follows that the same expressions of displacement bounds can be used for all the four rotations, except that $d_{T}$ must be replaced by $d_{C}$; 
hence the bound is:

$$
\begin{aligned}
& 2\left(\sin \left(\frac{\alpha_{\max }}{2}\right)+\sin \left(\frac{1}{2} \arcsin \left(\frac{2 \sin \left(\alpha_{\max } / 2\right)}{\cos \gamma_{\max }}\right)\right)\right)\left(d_{C}+l\right) \\
& +2 \sin \left(\beta_{\max } / 2\right)\left(l+d_{C}\right) \\
& +2 \sin \left(\frac{\cos \gamma_{\max }}{4 \cos \gamma_{\max }-2} \cdot|\Delta \theta|\right)\left(l \cdot \cos \left(\lambda_{\min } / 2\right)+d_{C}\right)
\end{aligned}
$$

\subsection{The sphere of the calf at the ankle joint}

This sphere undergoes the same rotations as the other sphere of the calf, but since we know that the whole transformation results in a translation of vector $\overrightarrow{\Delta L}$ of the center of the sphere, we can decompose the transformation into two motions: first a translation of vector $\overrightarrow{\Delta L}$, and then 4 consecutive rotations (of angles $\alpha_{1}, \alpha_{2}, \beta$ and $\delta \theta$ ) about axes passing through the center of the sphere. During any of these 4 rotations the distance of the points of the sphere to the rotation axis is at most $d_{C}$, and thus the following bound on the total displacement undergone by the points of the sphere follows:

$$
\begin{aligned}
& \|\overrightarrow{\Delta L}\|+2 \sin \left(\alpha_{\max } / 2\right) d_{C} \\
& +2 \sin \left(\frac{1}{2} \arcsin \left(\frac{2 \sin \left(\alpha_{\max } / 2\right)}{\cos \gamma_{\max }}\right)\right) d_{C} \\
& +2 \sin \left(\beta_{\max } / 2\right) d_{T}+2 \sin \left(\frac{\cos \gamma_{\max }}{4 \cos \gamma_{\max }-2} \cdot|\Delta \theta|\right) d_{C}
\end{aligned}
$$

\subsection{The sphere of the foot}

Since this sphere is attached to the end effector (the foot), the global transformation undergone is simple: a translation of vector $\overrightarrow{\Delta L}$ followed by a rotation of angle $\Delta \theta$ about a vertical axis passing through the center of the sphere. A simple bound of the displacements undergone by the points of the sphere can be deduced:

$$
\|\overrightarrow{\Delta L}\|+2 \sin \left(\frac{|\Delta \theta|}{2}\right) d_{F}
$$

Finally, by regrouping the bounds (27), (28), (29), (30), and (31), noticing that the bound (28) is greater than the bound (27), we obtain the global bound of Theorem 4.1, and that concludes the demonstration.

\section{Global bound}

We now combine the bounds obtained in the two previous sections. At an instant $t \in[0, T]$, we can describe the configuration of the robot lower body with the position of the CoM and orientation of the waist, and the position and orientation of both feet relatively to the CoM position and the waist orientation. For the half-step with input parameters $\left(S F_{x}(0), S F_{y}(0), S F_{\theta}(0)\right)$, it gives us the following values:

- For the CoM position and the waist orientation: $x(t), y(t)$, and $\frac{S F_{\theta}(t)}{2}$. 
- For the swing foot relative position:

$$
\underbrace{\left(\begin{array}{cc}
\cos \left(-S F_{\theta}(t) / 2\right) & -\sin \left(-S F_{\theta}(t) / 2\right) \\
\sin \left(-S F_{\theta}(t) / 2\right) & \cos \left(-S F_{\theta}(t) / 2\right)
\end{array}\right)}_{R_{-S F_{\theta}(t) / 2}}\left(\begin{array}{c}
S F_{x}(t)-x(t) \\
S F_{y}(t)-y(t)
\end{array}\right)
$$

- $\frac{S F_{\theta}(t)}{2}$ for the swing foot relative orientation.

- For the support foot relative position:

$$
R_{-S F_{\theta}(t) / 2}\left(\begin{array}{c}
-x(t) \\
-y(t)
\end{array}\right)
$$

- $\frac{-S F_{\theta}(t)}{2}$ for the support foot relative orientation.

Using the notations of section 3 , for the half-step with input parameters $\left(S F_{x}(0)+\Delta x, S F_{y}(0)+\right.$ $\left.\Delta y, S F_{\theta}(0)+\Delta \theta\right)$, those values are:

- For the CoM position and the waist orientation: $x^{\prime}(t), y^{\prime}(t)$, and $\frac{S F_{\theta}^{\prime}(t)}{2}$.

- For the swing foot relative position:

$$
R_{\left(-S F_{\theta}^{\prime}(t)+S F_{\theta}(t)\right) / 2} R_{-S F_{\theta}(t) / 2}\left(\begin{array}{c}
S F_{x}^{\prime}(t)-x^{\prime}(t) \\
S F_{y}^{\prime}(t)-y^{\prime}(t)
\end{array}\right)
$$

- $\frac{S F_{\theta}^{\prime}(t)}{2}$ for the swing foot relative orientation.

- For the support foot relative position:

$$
R_{\left(-S F_{\theta}^{\prime}(t)+S F_{\theta}(t)\right) / 2} R_{-S F_{\theta}(t) / 2}\left(\begin{array}{c}
-x^{\prime}(t) \\
-y^{\prime}(t)
\end{array}\right)
$$

- $\frac{-S F_{\theta}^{\prime}(t)}{2}$ for the support foot relative orientation.

As in section 4 , we call $\nu$ the vertical axis passing through the CoM. Let $r_{\max }$ be a bound of the distance between $\nu$ and any point of the robot lower body at all time: the robot lower body is always completely included in the cylinder of axis $\nu$ and radius $r_{\max }$. It follows that the displacements resulting from a rotation $R_{\psi}$ of axis $\nu$ are bounded by $2 \sin (|\psi| / 2) r_{\max }$. Using also the inequalities (7),(8),(9),(18),(19), we can show that at instant $t$, the distance between the two CoM positions is at most $\frac{\sqrt{\Delta x^{2}+\Delta y^{2}}}{2}=\frac{\|\overrightarrow{L L}\|}{2}$, and the difference between the two waist orientations is bounded by $|\Delta \theta| / 2$, while for both legs, the relative foot position is changed by at most $\|\overrightarrow{\Delta L}\|+2 \sin (|\Delta \theta| / 4) r_{\max }$ and the relative foot orientation by at most $|\Delta \theta| / 2$. A bound on the total displacement undergone by the physical points of the robot lower body is given by the sum of bounds for the two following displacements: first the displacement resulting from the modification of the CoM position and waist orientation, and then the displacement resulting from the modification of the relative configurations of the legs. The latter can be bounded using Theorem 4.1, and it leads us to our main result: 
Table 1: Numerical bounds with standard parameters

\begin{tabular}{l|r}
\hline $\begin{array}{l}N, \text { the number of points of the grid } \\
\text { covering the whole input space: }\end{array}$ & Corresponding margin: \\
\hline 1,000 & $0.371 \mathrm{~m}$ \\
10,000 & $0.209 \mathrm{~m}$ \\
100,000 & $0.121 \mathrm{~m}$ \\
$1,000,000$ & $0.072 \mathrm{~m}$ \\
$10,000,000$ & $0.043 \mathrm{~m}$ \\
$100,000,000$ & $0.025 \mathrm{~m}$ \\
\hline
\end{tabular}

Theorem 5.1. Assuming the restrictions concerning the values $d_{T}, d_{C}, d_{F}, \gamma_{\max }, \lambda_{\min }, \lambda_{\max }, r_{\max }$ previously defined in this paper, we have the following result:

Given two input vectors $\left(S F_{x}(0), S F_{y}(0), S F_{\theta}(0)\right) \in \mathcal{E}$ and $\left(S F_{x}(0)+\Delta x, S F_{y}(0)+\Delta y, S F_{\theta}(0)+\Delta \theta\right) \in$ $\mathcal{E}$, the Hausdorff distance between $\mathcal{S} \mathcal{V}\left(\left(S F_{x}(0), S F_{y}(0), S F_{\theta}(0)\right)\right)$ and $\mathcal{S} \mathcal{V}\left(\left(S F_{x}(0)+\Delta x, S F_{y}(0)+\right.\right.$ $\left.\left.\Delta y, S F_{\theta}(0)+\Delta \theta\right)\right)$ is bounded by:

$$
\frac{\|\overrightarrow{\Delta L}\|}{2}+\Omega+\mathcal{B}\left(\|\overrightarrow{\Delta L}\|+\Omega, \frac{|\Delta \theta|}{2}\right)
$$

where $\Omega=2 \sin \left(\frac{|\Delta \theta|}{4}\right) r_{\max }$

\section{$6 \quad$ Numerical estimates}

We defined standard values that should approximately correspond to the normal use of a humanoid robot with almost human dimensions: $d_{T}=d_{C}=0.15 \mathrm{~m}, d_{F}=0.20 \mathrm{~m}, l=0.30 \mathrm{~m}, r_{\max }=0.50 \mathrm{~m}, \lambda_{\min }=\frac{\pi}{3}$, $\lambda_{\max }=\pi-2 \frac{\pi}{180}, \gamma_{\max }=\frac{\pi}{4}$. We also fixed the limits of the input space: $S F_{x}(0) \in[-0.30 m,+0.30 m]$, $S F_{y}(0) \in[-0.30 m, 0 m]$, and $S F_{\theta}(0) \in\left[-\frac{\pi}{6}, \frac{\pi}{6}\right]$. With those values, a grid covering the whole input space with spacings $\delta x, \delta y$ and $\delta \theta$ has about $N=\frac{0.6}{\delta x} \frac{0.3}{\delta y} \frac{\pi / 3}{\delta \theta}$ points. If we use $\delta x=\delta y$, we have $\delta \theta=\frac{1}{N} \frac{0.6 \times 0.3}{\delta x^{2}} \frac{\pi}{3}$. Any point in the input space cannot be further than $\delta x \sqrt{2} / 2$ from a position in the $(x, y)$ plane of a point of the grid, and the angle cannot be further than $\delta \theta / 2$ from an angle of a point of the grid. Thus, a safe margin of error for the swept volumes is given by the bound of Theorem 5.1, using $\|\overrightarrow{\Delta L}\|=\delta x \sqrt{2} / 2$ and $|\Delta \theta|=\frac{1}{2} \frac{1}{N} \frac{0.6 \times 0.3}{\delta^{2}} \frac{\pi}{3}$. For a fixed $N$, we can find the value of $\delta x$ that leads to the smallest bound, i.e. the value that defines the grid with the "best balance" between $\delta x(=\delta y)$ and $\delta \theta$. We did it for different values of $N$ and Table 1 shows the margins of error obtained. We also tried with values specifically calculated for the robot HRP-2, and obtained comparable results. As shown in table 1, if we want to use a margin of $0.07 m$, about 1,000,000 points are needed.

This means that the following method would be theoretically possible for safe and fast footstep planning: 
1) for each input of a grid of 1,000,000 regularly spaced points (with the proper balance between $\delta$ and $\delta \theta$ ), precompute the corresponding swept volumes expanded by $0.07 m$, i.e. the set of points at distance at most $0.07 \mathrm{~m}$ from the actual swept volume; 2) use the whole continuous input space for footstep planning, and when collisions must be checked, instead of using the actually executed halfstep $\left(S F_{x}(0), S F_{y}(0), S F_{\theta}(0)\right)$, use the precomputed expanded swept volume of the nearest neighbor of $\left(S F_{x}(0), S F_{y}(0), S F_{\theta}(0)\right)$ among the 1 million points. The theoretical bound given by Theorem 5.1 ensures the soundness of the approach: if no obstacle intersects the precomputed expanded swept volume, then the actual half-step $\left(S F_{x}(0), S F_{y}(0), S F_{\theta}(0)\right)$ also avoids the obstacles.

Of course, the soundness is partially lost at the execution time since the robot has an unavoidable drift. Nevertheless, by quantifying the relation between the uncertainty on the input parameters and the uncertainty on the distance to collision, we obtained theoretical bounds that were never used in the field of humanoid robot footstep planning: conservative upper bounds on how much a small perturbation of the input parameters can affect the trajectories of the physical points of the robot.

\section{Conclusion}

For a humanoid robot with almost human dimensions, trying to keep a distance of about $0.07 \mathrm{~m}$ to the obstacles is arguably a reasonable behavior: above $0.07 \mathrm{~m}$, the obstacle avoidance capabilities start to sensibly decrease, and a configuration where the distance to the obstacles is less than $0.07 \mathrm{~m}$ can be considered potentially unsafe. Table 1 shows that with such a margin, we need to deal with about $1,000,000$ inputs in order to obtain a $100 \%$ safe generalization on the whole input space. With a standard computer, typical swept volume approximations take about 20 to 40s (see [13]) and would lead to an unreasonable computation time for the 1 million points; however, the use of parallel architectures (e.g. GPUs) could significantly reduce the global computation time and make it affordable. Besides, it is not compulsory to cover the whole input space: in [7] and [6], Chestnutt et al. showed that it is also interesting to consider a discrete action set with the possibility to slightly modify any of the fixed actions. This corresponds to a finite cloud of small neighborhoods in the input spaces, and our approach would enable us to deal with it with guarantees that don't exist yet in the literature.

With further analyses, it might also be possible to tighten the bound (e.g. taking into account the fact that the modifications of the foot relative positions are horizontal), therefore reducing the necessary number of points. In particular, if instead of the whole input space we choose to study only trajectories

in the vicinity of a reference trajectory, we can easily find better values for $\lambda_{\min }, \lambda_{\max }, \gamma_{\max }$. As a result the bound obtained is likely to be much tighter.

Finally, the approach presented in this paper might be useful for other applications as well:

- It could be applied to robot manipulators that have a 6-DOF structure similar to the one of the robot leg considered.

- For 3D avatars, as well as in some particular areas of robotics (e.g. robotic surgery), the uncertainty 
on the trajectories really executed is almost zero, and we don't have to fear the robot drift. Our bounds could thus bring guarantees at the execution time. In these areas, using learning algorithms is often not recommended because the good generalization to unseen inputs can never be completely taken for granted. But in this paper, we showed that in a non-trivial case the generalization can in fact be obtained with guarantees, so our approach might enlarge the palette of tools that can be used in some applications where unexpected collisions can have dramatic consequences.

\section{APPENDIX}

\section{A Bounds for $\alpha_{1}$ and $\alpha_{2}$}

Figure 7 illustrates the first phase of the leg motion, where the correct hip joint - ankle joint axis is set. It shows several equalities and inequalities. First, $\alpha$ being the angle between $\vec{L}$ and $\vec{L}+\Delta \vec{L}$ $(\alpha \in[0, \pi]), \kappa$, the displacement undergone by the ankle after the two first rotations, is such that $\kappa=2 l \sin (\lambda / 2) \cdot 2 \sin (\alpha / 2)$. Then, since $2 l \sin (\lambda / 2) \cdot \sin (\alpha)$ is the distance between the initial position of the ankle joint and the axis defined by $\vec{L}+\Delta \vec{L}$, we have: $2 l \sin (\lambda / 2) \cdot \sin (\alpha) \leq\|\overrightarrow{\Delta L}\|$. Furthermore, if

$\gamma$ denotes the angle between the vertical axis $\nu$ and $\overrightarrow{\Delta L}$, then the distance between the intermediate axis and the final position of the ankle joint is equal to $2 l \sin (\lambda / 2) \cos \gamma \cdot \sin \alpha_{2}\left(\alpha_{2} \in[0, \pi]\right)$, and it is necessarily less than than $\kappa$, thus:

$$
\begin{gathered}
2 l \sin (\lambda / 2) \cos \gamma \cdot \sin \alpha_{2} \leq 2 l \sin (\lambda / 2) \cdot 2 \sin (\alpha / 2) \\
\cos \gamma \cdot \sin \alpha_{2} \leq 2 \sin (\alpha / 2)
\end{gathered}
$$

Assuming $\frac{2 \sin (\alpha / 2)}{\cos \gamma} \leq 1$, and $\alpha_{2} \in\left[0, \frac{\pi}{2}\right]$ :

$$
\begin{gathered}
\alpha_{2} \leq \arcsin \left(\frac{2 \sin (\alpha / 2)}{\cos \gamma}\right) \\
\left|2 \sin \left(\alpha_{2} / 2\right)\right| \leq 2 \sin \left(\frac{1}{2} \arcsin \left(\frac{2 \sin (\alpha / 2)}{\cos \gamma}\right)\right)
\end{gathered}
$$

As it can be seen on the view from the side (Figure 7 ), $\kappa_{1}$, the displacement undergone by the ankle joint through the first rotation of angle $\alpha_{1}$ (with $\alpha_{1} \in[0, \pi]$ ), is equal to $2 l \sin (\lambda / 2) \cdot 2 \sin \left(\alpha_{1} / 2\right)$, and it can be shown that it is also less than $\kappa$ (this can be seen on the view from the front: the intermediate position of the ankle joint is, on the blue circle, the closest point to the inital position of the ankle joint), so we have:

$$
\begin{gathered}
2 l \sin (\lambda / 2) \cdot 2 \sin \left(\alpha_{1} / 2\right) \leq 2 l \sin (\lambda / 2) \cdot 2 \sin (\alpha / 2) \\
\left|2 \sin \left(\alpha_{1} / 2\right)\right| \leq 2 \sin (\alpha / 2)
\end{gathered}
$$

From the inequality $2 l \sin (\lambda / 2) \cdot \sin (\alpha) \leq\|\overrightarrow{\Delta L}\|$, and assuming $\alpha \in\left[0, \frac{\pi}{2}\right]$, we deduce:

$$
\alpha \leq \arcsin \left(\frac{\|\overrightarrow{\Delta L}\|}{2 l \sin \left(\frac{\lambda_{\min }}{2}\right)}\right)=\alpha_{\max }
$$




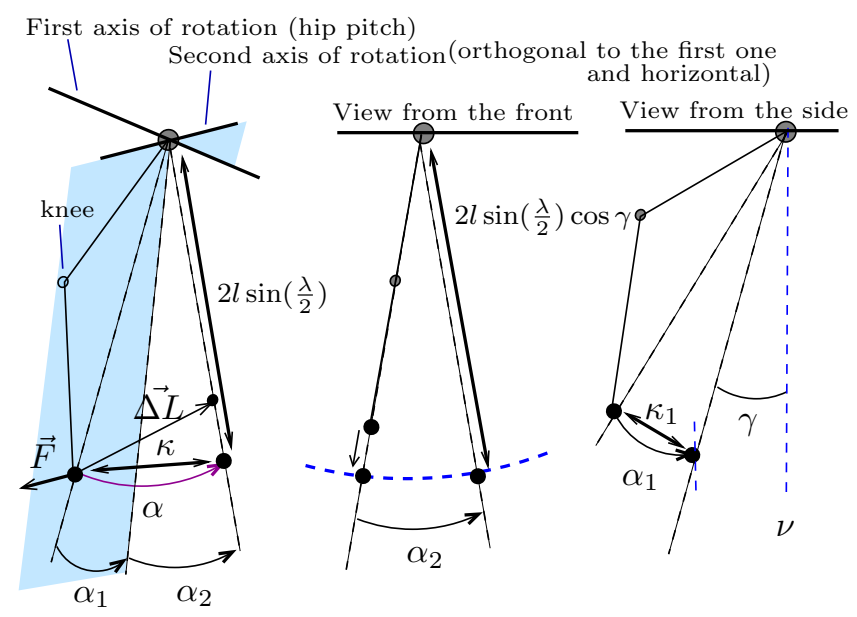

Figure 7: The two first rotations, to obtain the correct hip joint - ankle joint axis

And from the equations (36) and (38) it follows (again, we need to assume $\frac{2 \sin \left(\alpha_{\max } / 2\right)}{\cos \gamma_{\max }} \leq 1$ ):

$$
\begin{gathered}
\left|2 \sin \left(\alpha_{2} / 2\right)\right| \leq 2 \sin \left(\frac{1}{2} \arcsin \left(\frac{2 \sin \left(\alpha_{\max } / 2\right)}{\cos \gamma_{\max }}\right)\right) \\
\left|2 \sin \left(\alpha_{1} / 2\right)\right| \leq 2 \sin \left(\alpha_{\max } / 2\right)
\end{gathered}
$$

\section{B A bound for $\beta$}

During the second phase of the motion of the leg, pitch rotations are used to put the ankle joint at the correct position (see Figure 8). Typically, this sliding motion is the one that produces the largest displacements, by potentially moving the knee much more than the ankle. The positive or negative angle $\beta$ (variation of the hip pitch) is such that:

$$
2 l \sin \left(\frac{\lambda}{2}+\beta\right)=2 l \sin \left(\frac{\lambda}{2}\right)+\delta
$$

$\frac{\lambda}{2}+\beta$ stays in the range $\left[\frac{\lambda_{\min }}{2}, \frac{\lambda_{\max }}{2}\right] \subset\left(0, \frac{\pi}{2}\right)$, and therefore we have:

$$
\frac{\lambda}{2}+\beta=\arcsin \left(\sin \left(\frac{\lambda}{2}\right)+\frac{\delta}{2 l}\right)
$$

It follows:

$$
\beta=\int_{\sin (\lambda / 2)}^{\sin (\lambda / 2)+\delta /(2 l)} \frac{d t}{\sqrt{1-t^{2}}}
$$

We know: $\sin \left(\frac{\lambda}{2}\right)+\frac{\delta}{2 l} \in\left[\frac{\lambda_{\min }}{2}, \frac{\lambda_{\max }}{2}\right]$, and $|\delta| \leq\|\overrightarrow{\Delta L}\|$, so since $x \mapsto \frac{1}{\sqrt{1-t^{2}}}$ is an increasing function, we can deduce:

$$
|\beta| \leq \int_{\sin \left(\lambda_{\max } / 2\right)-\|\overrightarrow{\Delta L}\| /(2 l)}^{\sin \left(\lambda_{\max } / 2\right)} \frac{d t}{\sqrt{1-t^{2}}}
$$

Assuming that $\sin \left(\frac{\lambda_{\max }}{2}\right)-\frac{\|\overrightarrow{\Delta L}\|}{2 l} \geq-1$ :

$$
|\beta| \leq \underbrace{\frac{\lambda_{\max }}{2}-\arcsin \left(\sin \left(\frac{\lambda_{\max }}{2}\right)-\frac{\|\overrightarrow{\Delta L}\|}{2 l}\right)}_{\leq \pi}=\beta_{\max }
$$

It implies: $|2 \sin (|\beta| / 2)| \leq 2 \sin \left(\beta_{\max } / 2\right)$. 


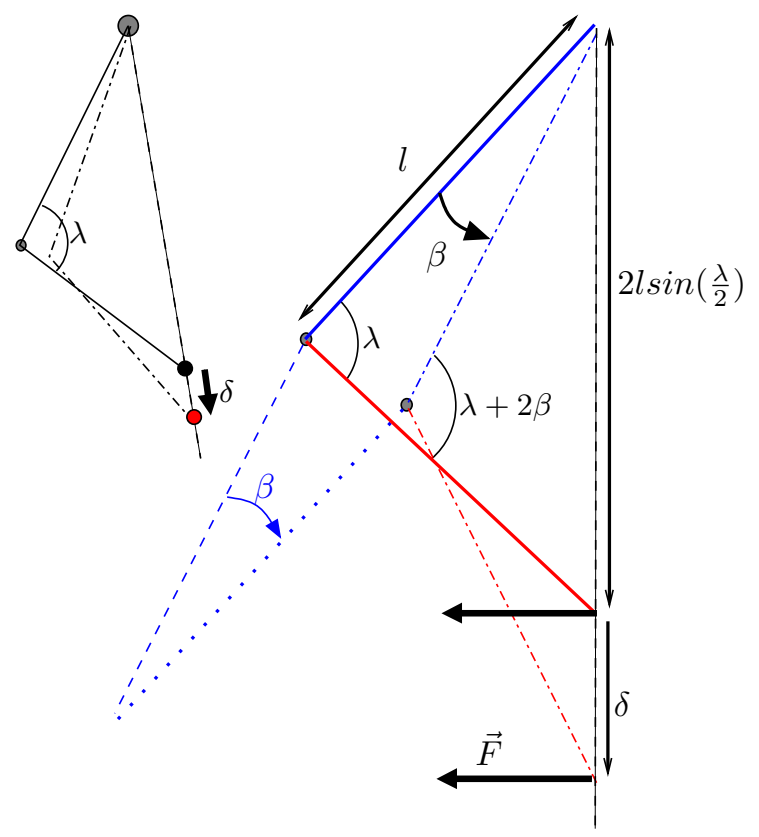

Figure 8: Sliding the ankle along the hip joint - ankle joint axis.

\section{A bound for $\delta \theta$}

After the second phase of the motion of the leg, the ankle is at the correct position and $\vec{F}$ is still unchanged, so what remains to be done is the modification of $\theta$. To modify $\theta$, we rotate the leg about the axis defined by $\vec{L}+\overrightarrow{\Delta L}$, and use the ankle pitch to keep the foot horizontal. Applying a rotation of angle $\delta \theta$ about this axis will not necessarily modify the foot yaw by $\delta \theta$. As shown on Figure 9 , let us consider the horizontal frame $\left(\overrightarrow{n_{1}}, \overrightarrow{n_{2}}\right)$ attached to the ankle joint, and such that $\overrightarrow{n_{1}}$ has the same yaw as $\vec{L}+\overrightarrow{\Delta L}$. We also call $\mathcal{P}$ the plane containing the ankle joint and orthogonal to $\vec{L}+\overrightarrow{\Delta L}$. During a rotation of angle $\delta \theta$ about the axis defined by $\vec{L}+\overrightarrow{\Delta L}$, the transformation applied to the foot can be decomposed into: 1) an orthogonal projection of $\vec{F}$ on the plane $\mathcal{P}$, then 2) a rotation of angle $\delta \theta$ about this axis, and finally 3 ) a projection along the direction defined by $\vec{L}+\overrightarrow{\Delta L}$ onto the horizontal frame $\left(\overrightarrow{n_{1}}, \overrightarrow{n_{2}}\right)$, followed by a resizing. If we denote by $\overrightarrow{n_{1}^{\prime}}$ the orthogonal projection of $\overrightarrow{n_{1}}$ on $\mathcal{P}$, and if $\vec{F}=x \cdot \overrightarrow{n_{1}}+y \cdot \overrightarrow{n_{2}}$, then after the projection on $\mathcal{P}$ it will be $x \cdot \cos \gamma \cdot \overrightarrow{n_{1}^{\prime}}+y \cdot \overrightarrow{n_{2}}$. Then, if we take $\left.\overrightarrow{\left(n_{1}^{\prime}\right.}, \overrightarrow{n_{2}}\right)$ as a basis of the plane $\mathcal{P}$, after the rotation of angle $\delta \theta$, the vector obtained will have the following coordinates:

$$
\left(\begin{array}{cc}
\cos \delta \theta & -\sin \delta \theta \\
\sin \delta \theta & \cos \delta \theta
\end{array}\right)\left(\begin{array}{c}
\cos \gamma \cdot x \\
y
\end{array}\right)=\left(\begin{array}{cc}
\cos \gamma \cdot \cos \delta \theta & -\sin \delta \theta \\
\cos \gamma \cdot \sin \delta \theta & \cos \delta \theta
\end{array}\right)\left(\begin{array}{l}
x \\
y
\end{array}\right)
$$

And after the projection back onto the plane generated by $\left(\overrightarrow{n_{1}}, \overrightarrow{n_{2}}\right)$, the vector $\overrightarrow{F^{\prime}}=x^{\prime} \cdot \overrightarrow{n_{1}}+\overrightarrow{y^{\prime}} \cdot \overrightarrow{n_{2}}$ will be obtained, with $x^{\prime}$ and $y^{\prime}$ such that:

$$
\left(\begin{array}{l}
x^{\prime} \\
y^{\prime}
\end{array}\right)=\left(\begin{array}{cc}
\frac{1}{\cos \gamma} & 0 \\
0 & 1
\end{array}\right)\left(\begin{array}{cc}
\cos \gamma \cdot \cos \delta \theta & -\sin \delta \theta \\
\cos \gamma \cdot \sin \delta \theta & \cos \delta \theta
\end{array}\right)\left(\begin{array}{l}
x \\
y
\end{array}\right)
$$



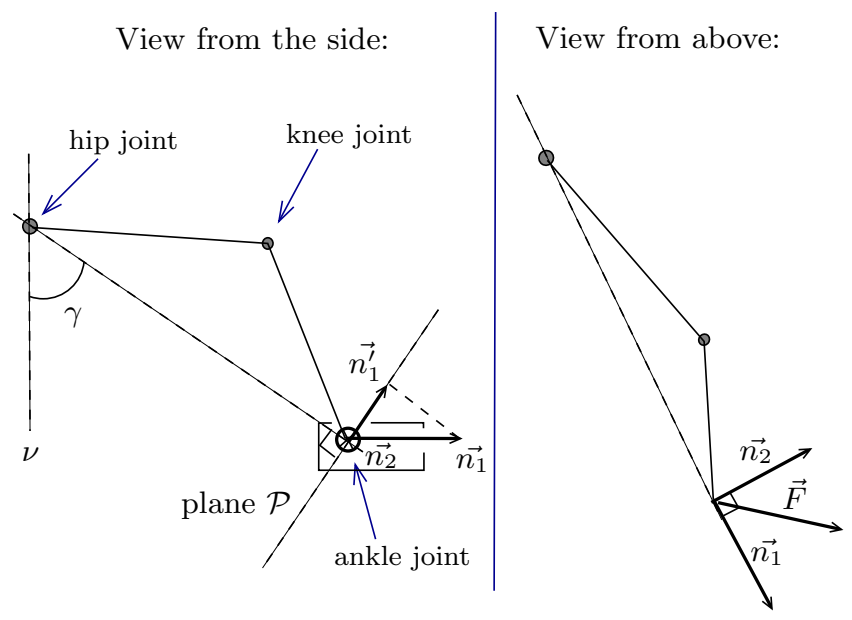

Figure 9: The frame $\left(\overrightarrow{n_{1}}, \overrightarrow{n_{2}}\right)$.

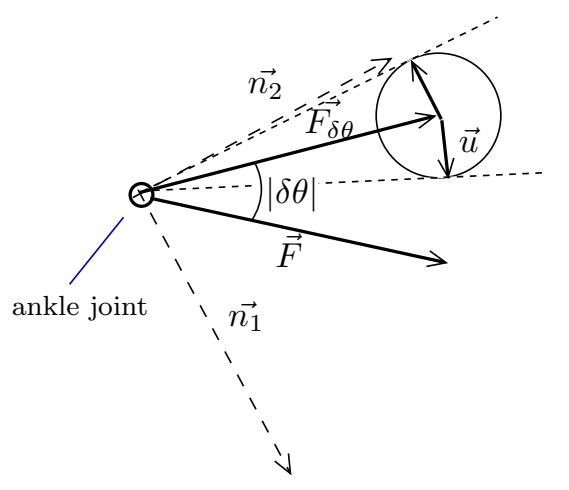

Figure 10: Finding an upper bound of the angle between $\vec{F}$ and $\overrightarrow{F_{\delta \theta}}$.

$$
\begin{aligned}
& \left(\begin{array}{l}
x^{\prime} \\
y^{\prime}
\end{array}\right)=\left(\begin{array}{cc}
\cos \delta \theta & \frac{-\sin \delta \theta}{\cos \gamma} \\
\cos \gamma \cdot \sin \delta \theta & \cos \delta \theta
\end{array}\right)\left(\begin{array}{l}
x \\
y
\end{array}\right) \\
& =\left(\begin{array}{cc}
\cos \delta \theta & -\sin \delta \theta \\
\sin \delta \theta & \cos \delta \theta
\end{array}\right)\left(\begin{array}{l}
x \\
y
\end{array}\right) \\
& +\sin \delta \theta \cdot\left(\begin{array}{cc}
0 & 1-\frac{1}{\cos \gamma} \\
\cos \gamma-1 & 0
\end{array}\right)\left(\begin{array}{l}
x \\
y
\end{array}\right)
\end{aligned}
$$

After the resizing, the new value of $\vec{F}$ will be $\frac{\vec{F}^{\prime}}{\left\|\vec{F}^{\prime}\right\|}$. Let us call $\overrightarrow{F_{\delta \theta}}$ the result of the rotation of $\vec{F}$ by angle $\delta \theta$ in the frame $\left(\overrightarrow{n_{1}}, \overrightarrow{n_{2}}\right)$. From the equation (49) we deduce that $\overrightarrow{F^{\prime}}=\overrightarrow{F_{\delta \theta}}+\vec{u}$, with:

$$
\begin{gathered}
\|\vec{u}\| \leq|\sin \delta \theta| \cdot \max \left(\left|1-\frac{1}{\cos \gamma}\right|,|\cos \gamma-1|\right) \\
\|\vec{u}\| \leq|\sin \delta \theta| \cdot \max \left(\left|1-\frac{1}{\cos \gamma}\right|,|\cos \gamma| \cdot\left|1-\frac{1}{\cos \gamma}\right|\right) \\
\|\vec{u}\| \leq|\sin \delta \theta| \cdot\left|1-(\cos \gamma)^{-1}\right|
\end{gathered}
$$

Since $\gamma<\frac{\pi}{3}, \cos \gamma>\frac{1}{2}$, and thus $0 \leq(\cos \gamma)^{-1}-1<1$, so $\|\vec{u}\|<1$. As a result (see Figure 10) the 
angle between $\overrightarrow{F_{\delta \theta}}$ and $\overrightarrow{F^{\prime}}$ is at most:

$$
\arcsin \left(|\sin \delta \theta| \cdot\left((\cos \gamma)^{-1}-1\right)\right)
$$

Since $\arcsin$ is convex on $[0,1]$, we also deduce that this value is bounded by $\left((\cos \gamma)^{-1}-1\right) \cdot \arcsin (\sin |\delta \theta|)$, and therefore also by:

$$
\left((\cos \gamma)^{-1}-1\right) \cdot|\delta \theta|
$$

It follows that, in order to obtain a rotation of angle $\Delta \theta$ in the frame $\left(\overrightarrow{n_{1}}, \overrightarrow{n_{2}}\right)$, the rotation about the axis defined by $\vec{L}+\overrightarrow{\Delta L}$ must be of angle $\delta \theta$ such that:

$$
|\delta \theta-\Delta \theta| \leq\left(\left(\cos \gamma_{\max }\right)^{-1}-1\right) \cdot|\delta \theta|
$$

And, as a result:

$$
|\delta \theta| \leq \frac{\cos \gamma_{\max }}{2 \cos \gamma_{\max }-1} \cdot|\Delta \theta|
$$

Assuming $\frac{\cos \gamma_{\max }}{2 \cos \gamma_{\max }-1} \cdot|\Delta \theta| \leq \pi$ :

$$
|2 \sin (|\delta \theta| / 2)| \leq 2 \sin \left(\frac{\cos \gamma_{\max }}{4 \cos \gamma_{\max }-2} \cdot|\Delta \theta|\right)
$$

\section{ACKNOWLEDGEMENTS}

This work was partially supported by a grant from the RBLINK Project, Contract ANR-08-JCJC-007501.

\section{REFERENCES}

[1] Y. Ayaz, K. Munawar, M. Bilal Malik, A. Konno, and M. Uchiyama. Human-like approach to footstep planning among obstacles for humanoid robots. In IEEE/RSJ Int. Conf. on Intelligent Robots and Systems (IROS'06), 2006.

[2] M. F. Beatty. Principles of Engineering Mechanics: Kinematics. Plenum Press, 1986.

[3] J.-M. Bourgeot, N. Cislo, and B. Espiau. Path-planning and tracking in a 3d complex environment for an anthropomorphic biped robot. In IEEE Intl. Conf. on Intelligent Robots and Systems, volume 3, pages 2509-2514, 2002.

[4] J. Chestnutt, J. Kuffner, K. Nishiwaki, and S. Kagami. Planning biped navigation strategies in complex environments. In IEEE Int. Conf. on Humanoid Robotics (Humanoids'03), 2003.

[5] J. Chestnutt, M. Lau, G. Cheung, J. Kuffner, J. Hodgins, and T. Kanade. Footstep planning for the honda asimo humanoid. In IEEE Int. Conf. on Robotics and Automation (ICRA'05), pages 631-636. 
[6] J. Chestnutt, P. Michel, K. Nishiwaki, J. Kuffner, and S. Kagami. An intelligent joystick for biped control. In IEEE Int. Conf. on Robotics and Automation (ICRA'06), pages 860 - 865, 2006.

[7] J. Chestnutt, K. Nishiwaki, J.J. Kuffner, and S. Kagami. An adaptive action model for legged navigation planning. In IEEE/RAS Int. Conf. on Humanoid Robotics (Humanoids'07), pages 196$202,2007$.

[8] J. Chestnutt, Y. Takaoka, M. Doi, K. Suga, and S. Kagami. Safe adjustment regions for legged locomotion paths. In IEEE Int. Conf. on Humanoid Robotics (Humanoids'10), 2010.

[9] M. Elmogy, C. Habel, and J. Zhang. Online motion planning for hoap-2 humanoid robot navigation. In IEEE/RSJ Int. Conf. on Intelligent Robots and Systems (IROS'09), 2009.

[10] J.-S. Gutmann, M. Fukuchi, and M. Fujita. Real-time path planning for humanoid robot navigation. In Int. Joint Conf. on Artificial Intelligence (IJCAI05), pages 1232-1237, 2005.

[11] T. Hasegawa, K. Nakagawa, and K. Murakami. Collision-free path planning of a telerobotic manipulator based on swept volume of teleoperated manipulator. In 5th IEEE Int. Symp. on Assembly and Task Planning, 2003.

[12] S. Kajita, F. Kanehiro, K. Kaneko, K. Fujiwara, K. Harada, and K. Yokoi. Biped walking pattern generation by using preview control of zero-moment point. In IEEE Int. Conf. on Robotics and Automation (ICRA'03), pages 1620-1626, 2003.

[13] Y.J. Kim, G. Varadhan, M.C. Lin, and D. Manocha. Fast swept volume approximation of complex polyhedral models. In 8th ACM symposium on Solid modeling and applications, pages 11-22, 2003.

[14] J. Kuffner, K. Nishiwaki, S. Kagami, M. Inaba, and H. Inoue. Footstep planning among obstacles for biped robots. In IEEE/RSJ Int. Conf. on Intelligent Robots and Systems (IROS'01), pages $500-505,2001$.

[15] N. Perrin, O. Stasse, L. Baudouin, F. Lamiraux, and E. Yoshida. Fast humanoid robot collision-free footstep planning using swept volume approximations. IEEE Trans. on Robotics, 28(2), 2012.

[16] N. Perrin, O. Stasse, F. Lamiraux, and E. Yoshida. A biped walking pattern generator based on "half-steps" for dimensionality reduction. In IEEE Int. Conf. on Robotics and Automation (ICRA'11), pages 1270-1275, 2011.

[17] N. Perrin, O. Stasse, F. Lamiraux, and E. Yoshida. Weakly collision-free paths for continuous humanoid footstep planning. In IEEE/RSJ Int. Conf. on Intelligent Robots and Systems (IROS'11), pages 4408-4413, 2011.

[18] F. Schwarzer, M. Saha, and J.-C. Latombe. Exact collision checking of robot paths. In 5th Workshop on the Algorithmic Foundations of Robotics (WAFR'02), 2002. 
[19] B. Siciliano and O. Khatib, editors. Springer Handbook of Robotics. 2008.

[20] M. Vukobratovic and B. Borovac. Zero-moment point - thirty five years of its life. Int. Journal of Humanoid Robotics, 1(1):157-173, 2004.

[21] E. Yoshida, I. Belousov, C. Esteves, and J.-P. Laumond. Humanoid motion planning for dynamic tasks. In IEEE/RAS Int. Conf. on Humanoid Robotics (Humanoids'05), pages 1-6, 2005. 